\title{
Measuring Time-Frequency Information and Complexity Using the Rényi Entropies
}

\author{
Richard G. Baraniuk,* Patrick Flandrin, $\diamond$ and Olivier Michel ${ }^{\diamond 1}$ \\ * Department of Electrical and Computer Engineering \\ Rice University \\ Houston, Texas, USA \\ - Laboratoire de Physique (URA 1325 CNRS) \\ Ecole Normale Supérieure de Lyon \\ 46 allée d'Italie, 69364 Lyon Cedex 07, France
}

\begin{abstract}
In search of a nonparametric indicator of deterministic signal complexity, we link the Rényi entropies to time-frequency representations. The resulting measures show promise in several situations where concepts like the time-bandwidth product fail.
\end{abstract}

\section{INTRODUCTION}

The term component is ubiquitous in the signal processing literature. Intuitively, a component is a concentration of energy in some domain, but this notion is difficult to translate into a quantitative concept. In fact, the concept of a signal component has never been - and may never be - clearly defined. In this paper, rather than address the question "what is a component?" directly, we investigate a class of quantitative measures of deterministic signal complexity and information content. While they do not yield direct answers regarding the locations and shapes of components, these measures are intimately related to the concept of a signal component, the connection being the intuitively reasonable supposition that signals of high complexity (and therefore high information content) must be constructed from large numbers of elementary components.

Our approach to complexity is based on entropy functionals and exploits the powerful analogy between deterministic signal energy densities and probability densities. For example, the Wigner time-frequency representation (TFR), $W_{s}(t, f)=\int s\left(u+\frac{\tau}{2}\right) s^{*}\left(u-\frac{\tau}{2}\right) e^{-j 2 \pi \tau f} d \tau$, which indicates the joint time-frequency content in a signal $s$, marginalizes to the time and frequency energy densities $\int W_{s}(t, f) d f=|s(t)|^{2}$ and $\int W_{s}(t, f) d t=|S(f)|^{2}$. The TFRs $C_{s}(t, f)$ of Cohen's class form an infinite set of generalizations of the Wigner TFR.

The probabilistic analogy evoked by the marginals suggests the Shannon entropy $H\left(C_{s}\right)=-\iint C_{s}(t, f) \log _{2} C_{s}(t, f) d t d f$ as a natural candidate for estimating the complexity of a signal through its TFR: The peaky TFRs of signals comprised of small numbers of elementary components would yield small entropy values, while the diffuse TFRs of more complicated signals would yield large entropy values. Unfortunately, however, the negative values taken on by the Wigner distribution and most other Cohen's class TFRs prohibit the application of the Shannon entropy due to the logarithm.

\section{The Rényi Entropies}

We propose to sidestep this negativity issue by employing the Rényi entropies $[1,2] H_{\alpha}\left(C_{s}\right)=\frac{1}{1-\alpha} \log _{2} \iint C_{s}^{\alpha}(t, f) d t d f$,

\footnotetext{
${ }^{1}$ Supported by NSF Grant MIP-9457438, ONR Grant N0001495-1-0849, Texas ATP Grant 003604-002, and URA 1325 CNRS.
}

which generalize the Shannon entropy to a family parameterized by $\alpha>0$. The resulting time-frequency information measure has a number of attractive properties. In addition to immunity to the negative TFR values that invalidate the Shannon approach [2], the third-order Rényi entropy measures signal complexity [1,2]: The information $H_{3}\left(C_{s}\right)$ in the TFR of the sum $s(t)=g(t)+g(t-T)$ of two separated signal components saturates (as $T \rightarrow \infty$ ) exactly one bit above the value $H_{3}\left(C_{g}\right)$ for a single component.

Our goal has been a detailed study of the properties and applications of these promising complexity measures, with emphasis on establishing a firm mathematical foundation. Interesting properties include the following [2]:

1. For integer orders $\alpha>1, H_{\alpha}\left(C_{s}\right)$ is defined for essentially all key TFRs, including even those distributions taking locally negative values.

2. For odd orders $\alpha>1, H_{\alpha}\left(C_{s}\right)$ is asymptotically invariant to TFR "cross-components" and therefore does not count them.

3. $H_{\alpha}\left(W_{s}\right)$ exhibits extreme sensitivity to phase differences between closely spaced components (ameliorated by time-frequency smoothing).

4. The range of $H_{\alpha}\left(W_{s}\right)$ values is bounded above and below. A single Gaussian pulse attains the lower bound, while "deterministic white noise" nears the upper bound.

5. The value of $H_{\alpha}\left(W_{s}\right)$ is invariant to arbitrary time and frequency shifts, scale changes, and shears and rotations in the time-frequency plane.

In recent work, we have applied the Rényi measures to random signals, introduced the notion of a Rényi dimension, and suggested how these measures can be employed to improve TFR performance through adaptivity.

Finally, we have introduced a new "Jensen-like" divergence measure [3]. While this quantity promises to be a useful indicator of the distance between two time-frequency distributions, it is currently limited to the analysis of positive definite TFRs. In spite of this rather severe limitation, this measure could prove useful for time-frequency based detection and recognition.

\section{REFERENCES}

[1] W. Williams, M. Brown and, A. Hero, "Uncertainty, Information, and Time-Frequency Distributions," SPIE 1566, 1991.

[2] P. Flandrin, R. Baraniuk, and $O$. Michel, "Time-Frequency Complexity and Information," Proc. IEEE ICASSP '94, 1994.

[3] O. Michel, R. Baraniuk, and P. Flandrin, "Time-Frequency Based Distance and Divergence Measures," Proc. IEEE Symp. Time-Frequency Analysis, 1994. 